\title{
Initialization of Unbalanced Radial Distribution Systems for Small Signal Stability Analysis
}

\author{
Javad Khazaei, Student Member, IEEE, Zhixin Miao, Senior Member, IEEE, Lakshan Piyasinghe, Student \\ Member, IEEE, Lingling Fan, Senior Member, IEEE
}

\begin{abstract}
This paper investigates the application of sweeping method in initializing the state variables of an unbalanced radial distribution system for small signal analysis. The unbalance is caused by a single-phase PV system. The system is composed of a three-phase induction machine (IM), a single-phase PV, a load, and a power factor correction (PFC) unit. The IM is modeled in positive-, negative-, and zero- sequence dynamic phasors and then converted into in a $a b c$ dynamic phasors frame. The PV, load and PFC and radial network are all modeled in phase-based dynamic phasors. The sweeping method along with embedded NewtonRaphson iterations is implemented to find the equilibrium point for initialization. The iterative procedures are illustrated and demonstrated by case studies. A dynamic simulation case study is also presented.
\end{abstract}

Index Terms-Dynamic Phasor (DP), Unbalance, Radial Distribution System, Induction Machine, Photovoltaic.

\section{INTRODUCTION}

To investigate the impact of increasing renewable energy resources on distribution networks, power system dynamics studies need to be carried out. These studies will be much more complicated when unbalance topology exists. Nonlinear dynamic simulation and small-signal analysis are the two main tools to study dynamic behavior. To carry out smallsignal analysis for an unbalanced system shown in Fig. 1, state variables are chosen to have constant steady-state values. Instantaneous currents and voltages are periodic at steadystate and cannot be used as the state variables [1]. On the other hand, complex Fourier coefficients (or dynamic phasors) of fundamental frequency component's phase $\mathrm{ABC}$ currents/voltages are constant at steady-state. Therefore, dynamic phasor-based modeling approach is suitable for small-signal analysis of unbalanced system [2,3].

To carry out small-signal analysis, operation conditions or equilibrium points need to be identified firstly through initialization. The dynamic-phasor based modeling approach has been presented in another paper by the authors [4]. The objective of this paper is to present a method to initialize a dynamic-phasor based model for an unbalanced system with single-phase PVs and three-phase induction machines (shown in Fig. 1).

Finding the steady-state values of the state variables is the initialization process. For the above mentioned system, there are two challenges to tackle for initialization:

Z. Miao, J. Khazaei, L. Piyasinghe and L. Fan are with Department of Electrical Engineering at University of South Florida, Tampa, FL (Email: zmiao@usf.edu).
- An efficient computing method for unbalanced radial distribution network power flow analysis is needed.

- Efficient initialization method of induction machine state variables is needed. The dynamic phasor-based induction machine model should take into consideration of unbalance effect.

In this paper, both challenges will be tackled.

Three-phase power flow analysis is usually carried out for unbalanced distribution networks [5]. For radial network, forward/backward sweeping method is usually adopted [6]. In the recent literature, improvement in power flow computing methods for unbalanced systems has been made. For example, $[7,8]$ proposed ways to enhance the convergence of power flow method for radial unbalanced systems when there are many distributed generation nodes. For research related to power flow, a solar panel or an induction machine is simplified as a PV or a PQ node. Initialization of the internal variables is not discussed.

There are other studies investigating the steady-state initial calculation of operating point conditions for induction machines or power electronic devices [9-11]. However, none of the mentioned references has considered the network.

In this paper, both aspects, the network and the devices, are considered. For the distribution network power flow analysis, sweeping method is applied. The dynamic phasor-based model of an induction machine including both positive and negative sequences is built and initialization is performed using Newton-Raphson method.

The paper is organized as follows. Section II describes the sweeping method implementation to the system in Fig. 1. Section III presents the induction machine model and the initialization procedure. Section IV presents case studies. Section V concludes the paper.

\section{SweEping Method ImPlementation}

\section{A. The Study System}

The power system which is used in this study is a unbalanced radial distribution system including an induction machine, Power Factor Correction (PFC) capacitors, a load and a single-phase PV. Fig. 1 shows the single-phase circuit diagram. All the elements are connected to the point of common coupling (PCC). Parameters of the system are given in the appendix. The single-phase PV is composed of an LCL filter for filtering out the unwanted harmonics. In single line diagram shown in Fig. 1, all the lines except for the PV 


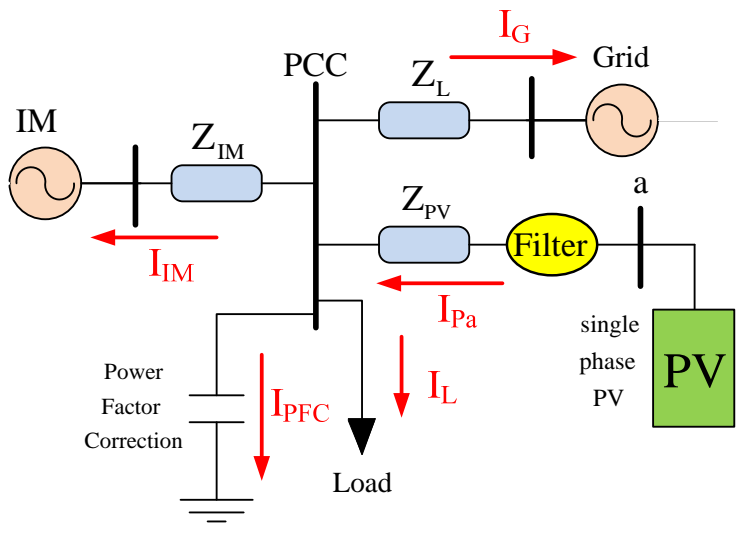

Fig. 1. The system under study. Load Power $=3000 W, \mathrm{PFC}=30 \mu F$. The line-to-line voltage level: $400 \mathrm{~V}$. Per-phase voltage: $230 \mathrm{~V}$.

are three phase balanced, and the PV line is a single phase line.

Most distribution systems are radial which enables the calculation of load flow starting from one point and moving forward/backward to the other points of the system. Usually, distribution network matrices are ill-conditioned with sparse bus admittance matrix. This can be justified by excessive number of nodes in systems compared with number of branches. Newton-Raphson or fast decoupled methods need to store the network admittance matrix and require computing power. On the other hand, sweeping method does not need to store the admittance matrix. In addition, fast decoupling methods can not applied when the ratio of $\frac{R_{\text {line }}}{X_{\text {line }}}$ is high. For a system in Fig. 1, sweeping method is more suitable.

\section{B. Sweeping method procedure}

Sweeping method starts from the initial guess of the PCC voltage. From the PCC voltage, and Newton-Raphson method, stator currents of the induction machine can be found. The line impedance $Z_{L 1}$ can be incorporated into the IM's stator impedance. Detailed description of Newton-Raphson method for IM in unbalanced conditions is given in the next section.

At the same time, with the first guess of PCC voltage, the PFC current and the load current will be calculated. The PV current will be also calculated based on reference PV power and initial PCC voltage using Newton-Raphson method. Fig. 2 gives the topology of the PV system where a LCL filter connected PV's converter to the grid. The PV panel and the interfacing converter have been aggregated as a block in Fig. 2 where the output is a sinusoidal ac voltage. Therefore, the $\mathrm{PV}$ and its converter are modeled as a voltage source, $V_{P b}$.

The following equations (in phasor domain) can be derived for the PV model presented in Fig. 2:

$$
\left\{\begin{array}{l}
V_{P C C}=I_{P a}\left(Z_{P V}+j \omega L_{a}\right)+V_{P C} \\
V_{P C}=V_{P b}-j \omega L_{b} I_{P b} \\
I_{P a}=I_{P b}-j \omega C V_{P C} \\
P_{\text {ref_PV }}=\Re\left(V_{P b} I_{P b}^{*}\right)
\end{array}\right.
$$

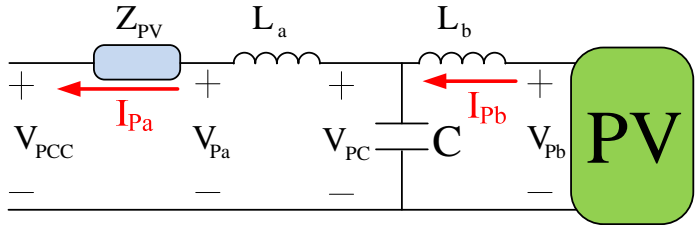

Fig. 2. The connection of PV and an LCL filter to the grid. $L_{a}=$ $0.01 H, L_{b}=0.02 H, C=2 \mu F$.

The reference PV power will be further expressed by $V_{P C C}$ and $I_{P a}$. Since $V_{P C C}$ is known, $I_{P a}$ can be found from the nonlinear equation using Newton-Raphson method.

Total current to the grid is then calculated by summing the PV current, PFC current, load current and IM currents. It should be noted that, as the PV is single-phase, currents for phase a is different from those of the two other phases. This will create unbalance in the PCC voltage and the grid current. The grid currents can be expressed as:

$$
\begin{aligned}
I_{G a} & =I_{P a}-I_{I M a}-I_{P F C a}-I_{L a} \\
I_{G b} & =-I_{I M b}-I_{P F C b}-I_{L b} \\
I_{G c} & =-I_{I M c}-I_{P F C c}-I_{L c}
\end{aligned}
$$

Assume that the grid voltage is fixed as $V_{G a}, V_{G b}, V_{G c}$, the PCC voltage will be updated using the calculated grid currents:

$$
V_{P C C}(\text { new })=\mathrm{V}_{\mathrm{Gabc}}+\mathrm{I}_{\mathrm{Gabc}} \mathrm{Z}_{\mathrm{L}}
$$

This new PCC voltage will be used to compute currents again until convergence. The flowchart of the sweeping algorithm is shown in Fig. 3.

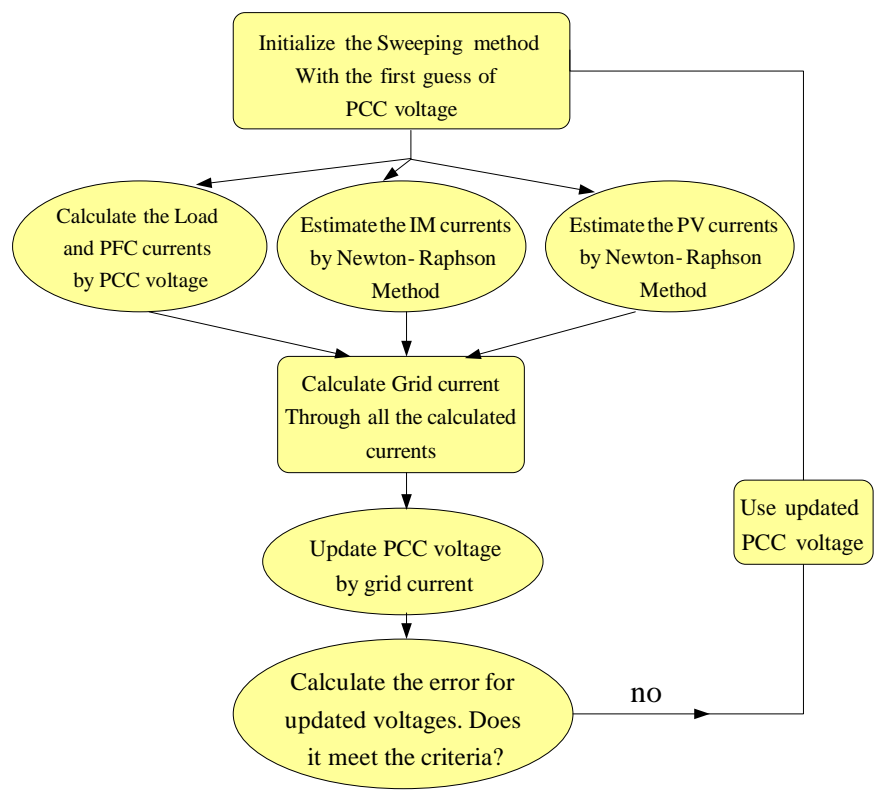

Fig. 3. Flowchart of the sweeping method.

\section{IndUCTION MACHINE MODEL AND INITIALIZATION}

In this section, initialization of an induction machine will be presented. The given information is the load torque $\left(T_{L}\right)$ 
and the PCC voltage. The PCC voltage is treated as the stator voltage of the IM when the line impedance $Z_{L 1}$ is aggregated into the stator impedance.

To capture the dynamic behavior of an induction machine under unbalance condition, the dynamic model should include both positive-, negative- and zero- (pnz)sequence components. The $p n z$ dynamic phasor-based dynamic model for unbalanced IM has been developed in [2]. This model will be used in our paper. All other elements, e.g., PV, PFC and the line, are all modeled by dynamic phasors in $a b c$ phase frame. Therefore, the pnz dynamic phasor-based IM model will be converted to $a b c$ dynamic phasor-based model as shown in Fig. 4.

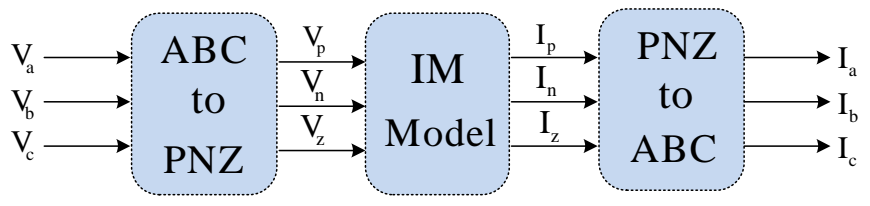

Fig. 4. Conversion from $a b c$ to $p n z$ and back to $a b c$ for an induction machine.

In the pnz reference frame, IM model's state variables include the stator and rotor currents: $I_{P s}, I_{N s}, I_{Z s}, I_{P r}, I_{N r}, I_{Z r}$, respectively. Subscripts $P, N, Z$ defines the positive, negative, and zero respectively where as subscripts $s$ and $r$ defines the stator and rotor signal respectively. Moreover, each signal has a real and imaginary part which will be shown by superscripts $r$ for real and $x$ for imaginary part of the signal. Neglecting the zero sequence components, eight current components $\left(I_{P s}^{r} I_{N s}^{r} I_{P r}^{r} I_{N r}^{r} I_{P s}^{x} I_{N s}^{x} I_{P r}^{x} I_{N r}^{x}\right)$ and three rotor speed components $\left(\omega_{r 0}, \omega_{r 2}^{r}\right.$ and $\left.\omega_{r 2}^{x}\right)$ should be initialized. The inputs of the IM are three-phase voltages of the stator in $a b c$ frame. The rotor voltages are considered to be zero. The stator voltages in $a b c$ should be converted to the $p n z$. The basic dynamic model of IM now is extended to $p n z$ reference frame and can be expressed by:

$$
V=C I+D \frac{d}{d t} I
$$

where, $V=\left[\begin{array}{llllll}V_{P s} & V_{N s}^{*} & 0 & V_{P r} & V_{N r}^{*} & 0\end{array}\right]^{T}$, and $I=$ $\left[\begin{array}{llllll}I_{P s} & I_{N s}^{*} & 0 & I_{P r} & I_{N r}^{*} & 0\end{array}\right]^{T}$. Matrices $C$ and $D$ then can be formulated as (5). Then state-space model can be found:

$$
\left\{\begin{array}{l}
\dot{I}=F_{x} \\
F_{x}=D^{-1} C I+D^{-1} V
\end{array}\right.
$$

where $F_{x}$ is the vector of derivative of induction machine currents including real and imaginary parts. Separating real and imaginary parts of $F_{x}$, eight equations of real and imaginary derivatives of IM currents will be achieved. Three equations of rotor speed in fundamental harmonic and second harmonic, presented in (7), can be added to shape the entire model in state space:

$$
\begin{aligned}
\omega_{r 0} & =\frac{1}{J}\left(P L_{m} \Im\left(I_{P s} I_{P r}^{*}+I_{N s}^{*} I_{N r}\right)-T_{L}-B \omega_{r 0}\right) \\
\omega_{r 2}^{r} & =\frac{1}{J} \Re\left(P L_{m} \frac{1}{2 j}\left(I_{P s} I_{N r}-I_{N s} I_{P r}\right)-\left(B+j 2 J \omega_{s}\right) \omega_{r 2}\right) \\
\omega_{r 2}^{x} & =\frac{1}{J} \Im\left(P L_{m} \frac{1}{2 j}\left(I_{P s} I_{N r}-I_{N s} I_{P r}\right)-\left(B+j 2 J \omega_{s}\right) \omega_{r 2}\right)
\end{aligned}
$$

where, $P$ is the number poles, $J$ is the inertia constant, $B$ is the damping factor, $\Re$ is the real part and $\Im$ is the imaginary part. Now, combining (7) and (8), an entire dynamic model of IM is obtained $\dot{X}=f(X)$,

where $X=\left[I_{P s}^{r} I_{N s}^{r} I_{P r}^{r} I_{N r}^{r} I_{P s}^{x} I_{N s}^{x} I_{P r}^{x} I_{N r}^{x} \omega_{r 0} \omega_{r 2}^{r} \omega_{r 2}^{x}\right]^{T}$, and $f(X)$ is the derivative of $X$.

The initialization will find the solution for $f(X)=0$. Newton-Raphson method starts with calculating the jacobian of $f(x)$. In the first step, initial guesses for the induction machine will be applied. In this paper, the initial guesses for all the parameters are set to zero except $\omega_{r 0}=188, I_{P s}^{r}=1$, and $I_{P r}^{r}=1$. The Newton's approach conducts the following iteration:

$$
X_{k}=X_{k-1}-\left.\left(\frac{\partial f}{\partial X}\right)^{-1}\right|_{X_{k-1}} f\left(X_{k-1}\right)
$$

where $X_{k-1}$ is the initial value in the last step. This iteration process will be continued till the error value is less than 0.001. After the error is converged, the initial parameters of the induction machine are obtained which will be used in sweeping method that was described in the previous section.

\section{CAse Study}

The model has been built in Matlab. The sweeping method along with two embedded Newton's approaches to find IM and PV initials have been applied. The IM model is built in $p n z$ reference frame, then it has been converted to $a b c$ frame, the grid is then modeled in $a b c$ and the single-phase PV is then added to the system in phase $a$. Two case studies have been conducted to examine the convergence of the algorithm for a balanced system (without PV) and an unbalanced system (with $2 \mathrm{~kW} \mathrm{PV).} \mathrm{Moreover,} \mathrm{dynamic} \mathrm{simulations} \mathrm{has} \mathrm{also} \mathrm{been}$ carried to demonstrate the system performance after a sudden torque change in the unbalanced system.

\section{A. Case 1: Balanced System}

In this case, the PV has been removed from the model and three-phase balanced voltages have been applied to the system. Convergence of the algorithm for the balanced case has been illustrated in Fig. 5 to Fig. 7. As it can be seen, the sweeping method for the entire system converges after five iterations which verifies the robustness of the algorithm in balanced condition.

Results of the line and IM RMS currents for the balanced case have been illustrated in Fig. 6. As expected, magnitudes for all three phases are the same for the balanced case. Results for rotor speed is illustrated in Fig. 7 which verifies the rotor speed does not have any second harmonic component due to balanced operation. 


$$
C=\left[\begin{array}{cccc}
r_{s}+j \omega_{s} L_{s} & 0 & j \omega_{s} L_{m} & 0 \\
0 & r_{s}-j \omega_{s} L_{s} & 0 & -j \omega_{s} L_{m} \\
j \omega_{s} L_{m}-j \omega_{r 0} \frac{P}{2} L_{m} & -j \omega_{r 2} \frac{P}{2} L_{m} & r_{r}+j \omega_{s} L_{r}-j \omega_{r 0} \frac{P}{2} L_{r} & -j \omega_{r 2} \frac{P}{2} L_{r} \\
-j \omega_{r 2}^{*} \frac{P}{2} L_{m} & -j \omega_{s} L_{m}-j \omega_{r 0} \frac{P}{2} L_{m} & -j \omega_{r 2}^{*} \frac{P}{2} L_{r} & r_{r}-j \omega_{s} L_{r}-j \omega_{r 0} \frac{P}{2} L_{r}
\end{array}\right]
$$
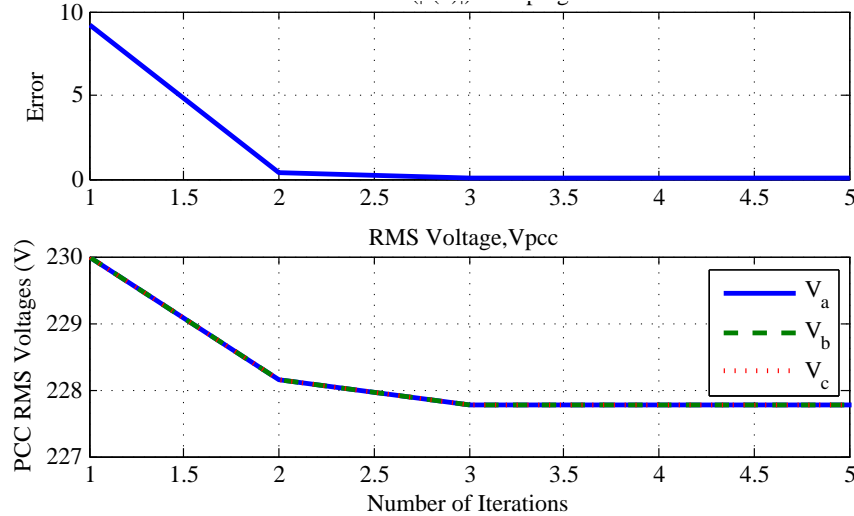

Fig. 5. PCC voltages in balanced case.
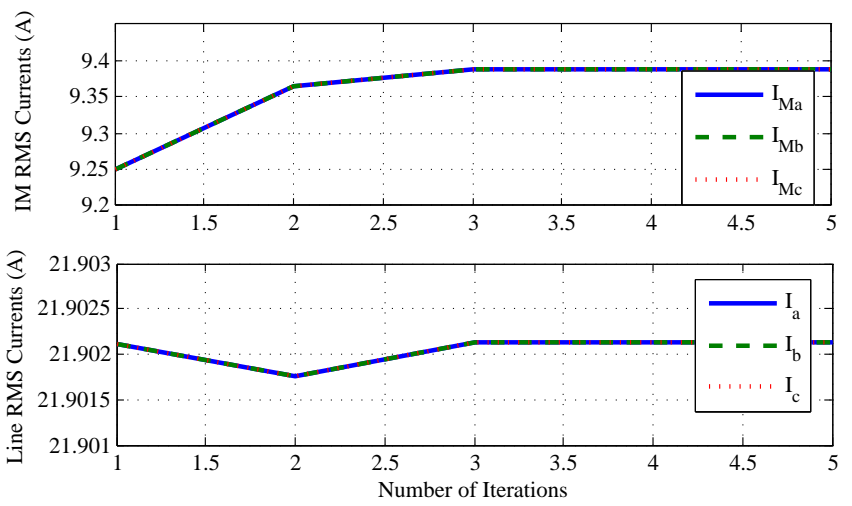

Fig. 6. Grid current and IM current.

\section{B. Case 2: Unbalanced System}

In this case, the system is faced with unbalance due to the connection of a single-phase PV at phase $a$. The power rating of the PV has been set to $2 \mathrm{~kW}$. The iterative results for the unbalanced system has been illustrated in Fig. 8 to Fig. 10. As it can be seen, the sweeping method in this case takes seven iterations to converge.

Fig. 9 shows that line current at phase $a$ is less than the other two phases which is caused by operation of a singlephase PV at phase a. Furthermore, Fig. 10 shows the rotor speed and its second harmonic components for unbalanced case. As it can be observed, by connecting the single phase $\mathrm{PV}$, the second harmonic components of the PV will have a
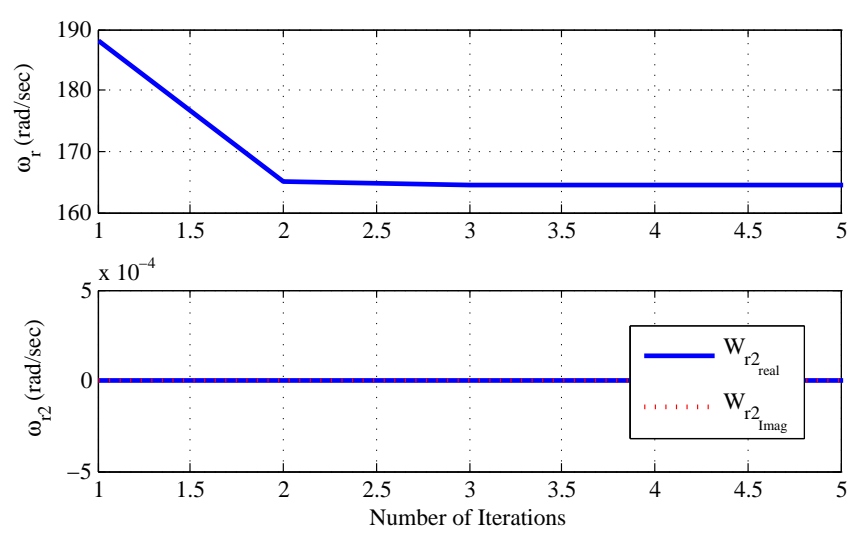

Fig. 7. rotor speeds in balanced case.

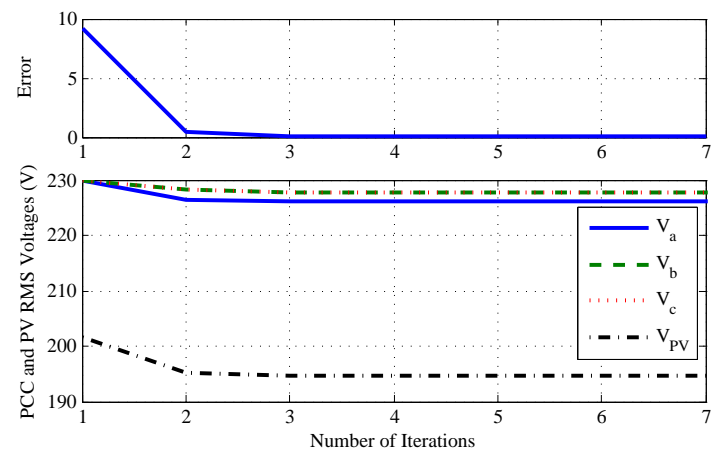

Fig. 8. Simulation results of sweeping method for voltages in unbalanced case

value and the sweeping method can obtain the initial values for these components as well.

\section{Case 3: Dynamic Simulation of an Unbalanced System}

In this part, the model is tested in a dynamic event. The model can be used for eigenvalue analysis after linearizing. Detailed parameters of the system have been given in the Appendix. The initial torque for the IM has been considered as 28 N.M. For the dynamic event, a torque change from 28 $N . M$ to 25 N.M will be applied to the system at time 10 sec. Results of simulation have been illustrated in Fig. 11. The initial calculation has guaranteed the flat run of the system as it can be observed. 


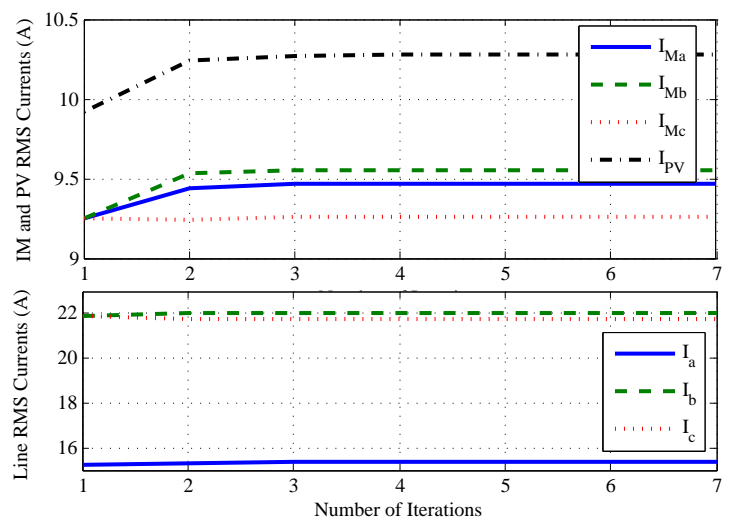

Fig. 9. Simulation results of sweeping method for currents in unbalanced case
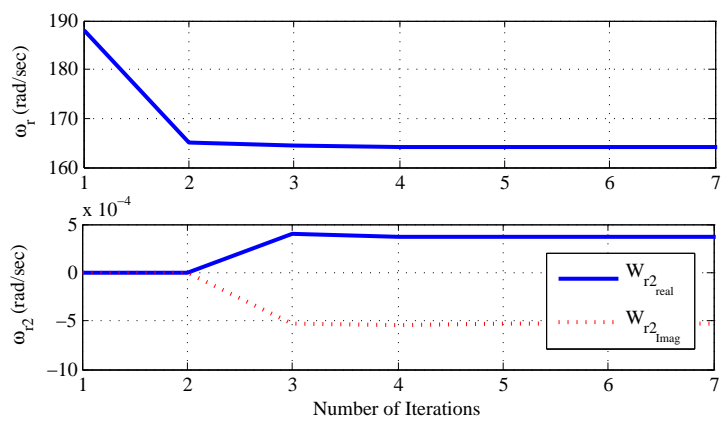

Fig. 10. Simulation results of sweeping method for rotor speed in unbalanced case

\section{CONCLUSION}

In this paper, sweeping method along with Newton-Raphson method is implemented to initialize the parameters of an unbalanced distribution system. The system is composed of a single phase PV, an Induction Machine, load, Power Factor Correction (PFC) unit and transmission lines. The single-phase PV introduces unbalance to the system. The IM is modeled by using the dynamic phasor concept in positive-negative-zero sequence. The state space model for entire microgrid is then derived. Given the initial guess for PCC voltage, NewtonRaphson method is used to initialize the parameters (including currents) of IM and PV, then sweeping method is initiated to go back and forth to update the PCC voltage. In each step of sweeping method, the embedded Newton-Raphson iterations will be carried out to find IM and PV parameters. Case studies show that the proposed initialization approach can find the initial values of the system in unbalanced conditions.

\section{APPENDIX}

\section{REFERENCES}

[1] J. Sun, "Small-signal methods for ac distributed power systems-a review," Power Electronics, IEEE Transactions on, vol. 24, no. 11, pp. $2545-2554,2009$.

[2] A. M. Stankovic, S. R. Sanders, and T. Aydin, "Dynamic phasors in modeling and analysis of unbalanced polyphase ac machines," Energy Conversion, IEEE Transactions on, vol. 17, no. 1, pp. 107-113, 2002.

[3] P. Mattavelli, A. M. Stankovic, and G. C. Verghese, "Ssr analysis with dynamic phasor model of thyristor-controlled series capacitor," Power Systems, IEEE Transactions on, vol. 14, no. 1, pp. 200-208, 1999.
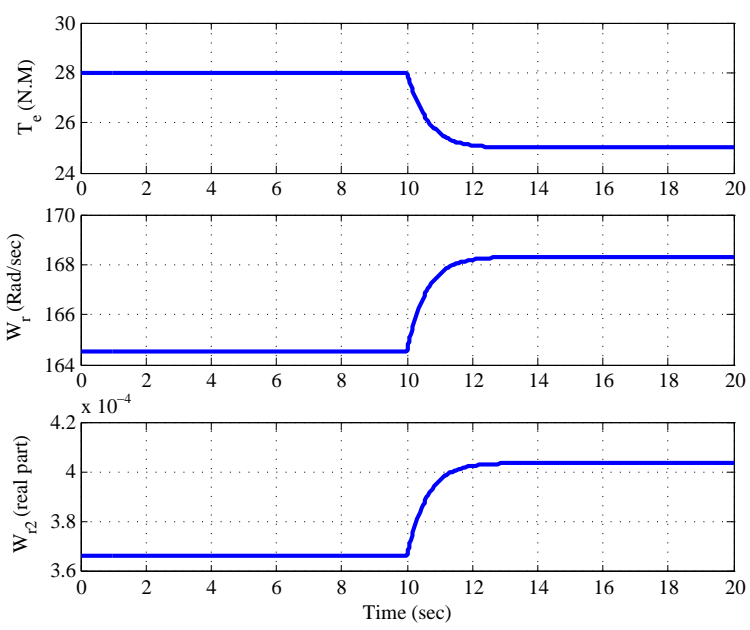

Fig. 11. Dynamic simulation of system with torque change.

TABLE I

PARAMETERS OF INDUCTION GENERATOR

\begin{tabular}{|c|c|}
\hline Total capacity & $5.5 \mathrm{kVA}$ \\
Nominal voltage & $400 \mathrm{~V}$ \\
Frequency & $60 \mathrm{~Hz}$ \\
$R_{s}$ & $2.52 \Omega$ \\
$R_{r}$ & $2.67 \Omega$ \\
$X_{l s}$ & $3.39 \Omega$ \\
$X_{l r}$ & $3.39 \Omega$ \\
$X_{M}$ & $197 \Omega$ \\
$J$ & $0.486 \mathrm{~kg} . \mathrm{m}^{2}$ \\
$P($ poles $)$ & 4 \\
\hline
\end{tabular}

[4] Z. Miao, L. Piyasinghe, J. Khazaei, and L. Fan, "Dynamic phasor-based modeling of unbalanced radial distribution systems," Power Systems, IEEE Transactions on, 2015.

[5] C. S. Cheng and D. Shirmohammadi, "A three-phase power flow method for real-time distribution system analysis," Power Systems, IEEE Transactions on, vol. 10, no. 2, pp. 671-679, 1995.

[6] D. Das, H. Nagi, and D. Kothari, "Novel method for solving radial distribution networks," IEE Proceedings-Generation, Transmission and Distribution, vol. 141, no. 4, pp. 291-298, 1994.

[7] Y. Ju, W. Wu, B. Zhang, and H. Sun, "An extension of fbs three-phase power flow for handling pv nodes in active distribution networks," Smart Grid, IEEE Transactions on, vol. 5, no. 4, pp. 1547-1555, 2014.

[8] H.-D. Chiang, T.-Q. Zhao, J.-J. Deng, and K. Koyanagi, "Homotopyenhanced power flow methods for general distribution networks with distributed generators," Power Systems, IEEE Transactions on, vol. 29, no. 1, pp. 93-100, 2014.

[9] J. F. M. Padrón and A. E. F. Lorenzo, "Calculating steady-state operating conditions for doubly-fed induction generator wind turbines," Power Systems, IEEE Transactions on, vol. 25, no. 2, pp. 922-928, 2010.

[10] N. Malik and S. Haque, "Steady state analysis and performance of an isolated self-excited induction generator," Energy Conversion, IEEE Transactions on, no. 3, pp. 134-140, 1986.

[11] C. Fuerte-Esquivel, E. Acha, and H. Ambriz-Perez, "A comprehensive newton-raphson upfc model for the quadratic power flow solution of practical power networks," Power Systems, IEEE Transactions on, vol. 15 , no. 1 , pp. 102-109, 2000.

TABLE II

DATA OF THE LINES IN NETWORK

\begin{tabular}{|c|c|c|c|}
\hline Line No & Line Type & $\mathrm{Z}(\Omega / \mathrm{km})$ & Length $(\mathrm{m})$ \\
\hline 1 & Grid Line (3-phase) & $0.284+\mathrm{j} 0.0825$ & 105 \\
2 & IM Line (3-phase) & $0.497+\mathrm{j} 0.0861$ & 105 \\
3 & PV Line (single-phase) & $0.0144+\mathrm{j} 0.0064$ & 30 \\
\hline
\end{tabular}

